\title{
HD151018: strong magnetic field in a giant O-type star?
}

\author{
M. S. Rubinho, A. Damineli, A. Carciofi and D. Moser \\ Instituto de Astronomia, Ciências Atmosféricas e Geofísica, Universidade de São Paulo
}

\begin{abstract}
HD151018 is a normal O-type giant with periodic variability of the wind seen in both spectroscopy and linear polarimetry. The characteristics of the wind emission strongly indicate a magnetically confined wind. This work presents the observational results and an initial modelling of the polarimetric modulation of this star.
\end{abstract}

Keywords. magnetic fields, polarimetric, spectroscopic.

While there are many B-type stars with known magnetic fields, the number drops to less than a dozen when it comes to O stars (Wade \& MiMeS, 2015). HD 151018 is a O-type giant star with wind emission, which strongly indicates a wind modulated by the magnetic field (confining here has a very specific meaning eg., Ud-Doula, 2008). The aim of this work is to report our observations of the polarimetric modulation of HD 151018. Also, to get insights of the magnetic wind confinement configuration in this star. HD 151018 was monitored both spectroscopically and polarimetrically over the last few years at the OPD/LNA observatory in Brazil. The $H_{\alpha}$ emission profiles resembles others similar O stars with magnetic field. The Fig.1a shows a phase-locked line profile variations in $H_{\alpha}$ with a period of about $4.3 \mathrm{~d}$. The Fig.1b presents the results of linear polarization which shows a data variation with amplitude of $\geqslant 0.02 \%$ and a period of $\approx 4.5 \mathrm{~d}$. We also present our first findings on the application of physically motivated model (PMM), for polarimetry, which consists of two blobs of gas connected by a disk, as created by Carciofi et al. (2013). Although the PMM model is preliminary, the model reproduces qualitatively well the polarimetric curve.

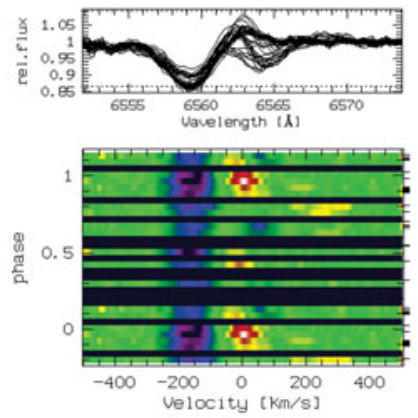

(a)

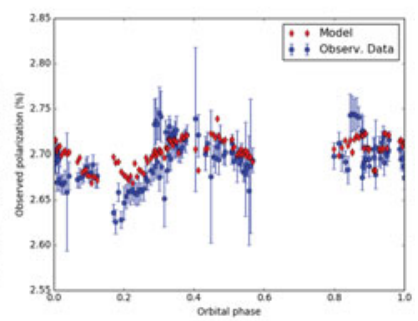

(b)

Figure 1. Observational data of HD151018 Right top: superposed normalized H $\alpha$ spectra. Right bottom: $\mathrm{H} \alpha$ dynamical spectrum. Left: Polarimetric data and the proposed preliminar model.

\section{References}

Wade, G. A., MiMeS Collaboration 2015, ASPCS, 494, 30

Carciofi, A., Faes, D., Townsend, R., \& Bjorkman, J. 2013, ApJ, 766, L9

Ud-Doula, A. 2008, Clumping in Hot-Stars Winds, 125 